\title{
LA UNIVERSIDAD PÚBLICA VENEZOLANA: UNA SÍNTESIS RECIENTE
}

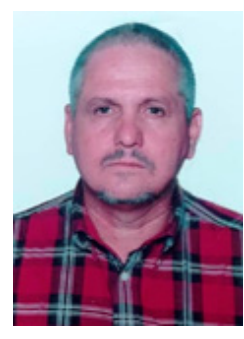

Fecha de recepción:16/05/2020
Fecha de revisión:24/05/2020

Carlos Eduardo Blanco

Facultad de Humanidades y Educación

Universidad Central de Venezuela

carlos.e.blanco@ucv.ve

ORCID 0000-0002-0175-247X

\section{RESUMEN}

El presente ensayo expresa un ejercicio investigativo que tiene como propósito esencial explorar críticamente un aspecto de la realidad para aproximarse a un fenómeno social particular como lo es la universidad estatal venezolana como institución compleja. Se trata de una evaluación crítica de investigaciones previas relativas a dicha institución para un contexto geográfico en particular. Busca contribuir al estado del conocimiento de este objeto de estudio al identificar relaciones, contradicciones o inconsistencias, así como proponer posibles soluciones. Tema de altísima importancia social en los países latinoamericanos, la institución universitaria y aspectos de la manera como se han conducido estas instituciones incluyen nudos críticos de interés. Un problema de investigación evidenciado ha sido la escasez de referencias integradoras y comprensivas recientes acerca de la institución universitaria en Venezuela. El presente trabajo tuvo como objetivo central reunir, analizar e integrar críticamente aspectos salientes del devenir de la universidad venezolana reciente. Mediante fuentes relevantes se abordaron rasgos y fenómenos destacables en las instituciones universitarias de dicho país, con énfasis en la conducción que ellas han tenido tanto contemporáneamente como hoy en día. Se evidenció que la universidad venezolana ha mantenido en general un desarrollo histórico típico de la universidad crítica latinoamericana, similarmente a sus pares de la región. No obstante, las universidades de este país se caracterizan actualmente por atravesar tiempos de muy serias e inéditas dificultades.

Palabras clave: Universidades, Educación Superior, Venezuela. 


\title{
PUBLIC UNIVERSITIES IN VENEZUELA: A CURRENT SYNTHESIS
}

\begin{abstract}
This essay is an investigative attempt whose essential purpose is to explore critically an aspect of reality in order to approach a particular social phenomenon such as the Venezuelan public university as a complex institution. It is a critical evaluation of previous research related to that institution for a particular geographic context. The essay seeks to contribute to the state of knowledge of its object of study by identifying relationships, contradictions or inconsistencies, as well as proposing possible solutions. That said, universities can be considered among the most important institutions in Latin America. Aspects of the way these institutions have been conducted include various critical nodes of interest worth-analyzing. A research problem in this regard has been the relative scarcity of integrative, comprehensive and recent references about universities in Venezuela. This investigation had as its central objective to compile, analyze and critically integrate various salient aspects of the Venezuelan universities in recent times. By means of relevant sources some important features and phenomena of these institutions in this country were approached, with an emphasis on the management that they have had both contemporarily and also currently. The analysis shows that Venezuelan universities have had in general a historical development similar to their critical Latin American counterparts. However, Venezuela's universities are currently characterized by going through a period of very serious and unprecedented difficulties.
\end{abstract}

Key words: Universities, Higher Education, Venezuela.

\section{INTRODUCCIÓN}

(...) una vez rebasado el exclusivo ámbito de la educación escolar, nos enfrentamos al problema del poder, de los conflictos de clase, del control del orden social en que está inmersa la universidad y al papel político ideológico que esta juega en la sociedad" (Aguirre Lora, 1997, p.11).

En el proceso de la educación venezolana, las universidades públicas de este país forman parte de varios aspectos centrales y complejos, merecedores de atención (Albornoz, 2006; Bifano, 2009; Parra-Sandoval, 2008; Rivas, 2014). Ejemplos de aspectos problemáticos de la educación universitaria de este país lo constituyen los eventos de orden político, social, legislativo, entre otros, ocurridos en los últimos años y que se relacionan con necesarios cambios en estas instituciones dentro de su condición de autonomía (Inciarte, García-Guadilla y Rama, 2011; Mendoza Angulo, 
2011). Tales eventos se incluyen en la no nueva, aunque ahora en el siglo XXI más pronunciada, pugna entre el Estado, con los sucesivos gobiernos, y estas casas del saber (Koudeir, 2019). En el presente ensayo se expone un análisis que busca dar una visión actualizada, crítica, de estas cuestiones, con énfasis en la conducción o gerencia académica que han venido teniendo las universidades venezolanas con relación a las funciones que deben llevar a cabo en beneficio de la sociedad. En cuanto al origen de este trabajo, él forma parte del proyecto de tesis doctoral "El artículo de investigación en revistas de Educación de Colombia y Venezuela”, proyecto D052517516, Doctorado en Educación, Comisión de Estudios de Postgrado, Facultad de Humanidades y Educación de la Universidad Central de Venezuela.

\section{LA UNIVERSIDAD VENEZOLANA. ASUNTO SOCIAL Y POLÍTICO.}

En el devenir de la educación nacional, la universidad venezolana se inició a partir de un seminario religioso en la primera mitad del siglo XVIII, con la Real y Pontificia Universidad de Caracas. Esta universidad sería Alma Mater de las demás instituciones del país, fundadas a partir de la segunda mitad del siglo XIX. Según Villarroel (1990), tras sus inicios en 1721 la importancia científica de la universidad de Caracas era nula, con poca o ninguna repercusión en el desarrollo del país. Ella gozaba de la misma autonomía de la universidad española antes de la invasión napoleónica que impondría el modelo francés. En 1827 Simón Bolívar le restauró la autonomía a la universidad, pero en lo organizativo y administrativo la institución continuó, tal vez en mayor grado, como napoleónica. En los siglos XIX y XX la universidad, ya republicana, de manera similar a las restantes de Latinoamérica, toma el rol principal de certificadora o tituladora de doctores, principalmente abogados. Al igual que el resto, era una institución que no investigaba, pues la poca investigación que en el país se hacía era por cuenta de personalidades, algunas de la cuales impartían clases en la universidad (Villarroel, 1990). La primera asignatura específicamente de investigación en toda la Universidad Central y en Venezuela se creó en la Facultad de Medicina, en 1925 (Blanco, 2007).

Tras el primer cuarto del siglo XX, circularon en Venezuela las ideas del Movimiento o Reforma de Córdoba, en buena parte a través de la denominada Generación de 1928 en Caracas. Así comenzó el tradicional enfrentamiento entre el Estado y la universidad (Acevedo Tarazona y Correa Lugos, 2018; Tunnermann, 1983; Villarroel, 1990) lo cual ha signado buena parte del carácter de la institución universitaria venezolana contemporánea. En ese ambiente, más tarde los estudios de postgrado en el Alma Mater de este país se inauguraron en 1941 en el área de Medicina, con la especialización de médicos higienistas, seguida por psiquiatría, venereología, tisiología, puericultura y pediatría, para reforzar la misión de la universidad de fomentar la ciencia en sus aspectos de investigación y aplicación (Bifano, 2009). En 1975 apareció el primer Catálogo

ORATORES ISSN Impreso: 2410-8928 ISSN Electrónico: L-2644-3988 Año 8. Número 12. Junio - Noviembre 2020 
General de Cursos de Postgrado y el Reglamento de Estudios para Graduados en dicha universidad. Estos programas fueron más bien producto de la iniciativa personal de profesores con formación de cuarto nivel en universidades de Europa y Estados Unidos, quienes asumieron el compromiso de desarrollar sus disciplinas a imagen del modelo que conocieron durante sus estudios en el extranjero. Una de las características de la comunidad universitaria venezolana ha sido la de no querer ser evaluada, ello debido posiblemente a que el ejercicio evaluativo se percibe con un carácter punitivo y fiscalizador que pudiera atentar contra una libertad o autonomía de cátedra no siempre bien entendidas (Bifano, 2009).

Con relación a una función principalmente política, a partir de la segunda década del siglo XX el movimiento estudiantil venezolano condujo a la universidad a ser un centro de lucha política y social en el país, matriz del movimiento político contemporáneo, con cuestionamiento del subdesarrollo y la dependencia, con planteamientos a favor de una sociedad más desarrollada y moderna. Unas tres décadas más tarde, en el ambiente de la Revolución Cubana de 1959, la universidad se comprometía más con los movimientos políticos y los propósitos de transformación social, a los cuales se incorporaron estudiantes y profesores. Luego, a partir de comienzos de los 1970, la derrota y pacificación de los grupos insurreccionales procastristas coincidieron y se relacionaron con una universidad que tendía a asumir el concepto de autonomía en gran medida formal, como soberanía casi exclusivamente territorial. El Estado venezolano creó entonces un grupo de universidades no autónomas (experimentales) y también otras tecnocráticas (Universidad de Oriente, Universidad Simón Bolívar) ideadas para promover mayor colaboración con el Estado, así como para colocar un contrapeso frente a las universidades autónomas, casi siempre opositoras radicales. Así, las etapas históricas aquí descritas evidencian tres tendencias centrales en el comportamiento tradicional de la universidad venezolana. Primero, poca o ninguna participación en los proyectos de desarrollo nacional. Segundo, oposición o diferenciación respecto de las políticas del Estado. Tercero, aislamiento del Estado y de la sociedad (Villarroel, 1990).

Con referencia a la función de investigación en la universidad venezolana, la institución pareció conservar la separación de la universidad francesa entre docencia e investigación, con dificultad para institucionalizar esta función hasta después de la caída del dictador Marcos Pérez Jiménez en enero de 1958. A partir de entonces se introdujo explícitamente la investigación en la Ley de Universidades y se crearon los Consejos de Desarrollo de cada institución (Bifano, 2008), organismos muy importantes que han solido ser el casi único apoyo financiero de los proyectos científicos y humanísticos, así como sus actividades relacionadas, dentro de las altas casas del saber. A partir de 1958 la casi totalidad de la investigación que se ha realizado en el país, aparte del estatal Instituto Venezolano de Investigaciones Científicas, se ha venido llevando a cabo en 
las universidades estatales autónomas. Así, hasta bien entrada la década de los 1980 se reportó un crecimiento en tal función (Villarroel, 1990) lo cual ha contrastado con su notable decaimiento en tiempos recientes (Aguado-López y Becerril-García, 2016; Ramírez y Salcedo, 2016; Van Noorden, 2014). Pero según Villarroel (1990) la investigación que tradicionalmente han realizado las universidades venezolanas no ha tenido mayor incidencia sobre el desarrollo socioeconómico del país. Esto probablemente debido a que se ha tratado de una investigación mayormente académica, intramuros, exenta de pragmatismo social, en un contexto en que los factores de poder del país frecuentemente han concebido a las universidades como centros de despilfarro de recursos públicos, cuando no refugio de subversivos.

Entonces, la labor investigativa universitaria del país rara vez se ha insertado en proyectos nacionales. Los problemas de investigación abordados poco frecuentemente han nacido de necesidades sociales, sino más bien de preocupaciones intelectuales, personales de los profesores. Incluso la Ley de Universidades misma ha planteado colaborar en la orientación y esclarecimiento de los problemas nacionales (Ley de Universidades, 1970), como especie de oráculos, pero que casi nunca han sido consultadas, hoy en día mucho menos. Por lo tanto, la investigación universitaria venezolana ha solido carecer de efectividad científica, se producen investigaciones, se publican textos, pero se genera muy poco conocimiento. Así, se ha tenido una función de investigación mitificada, esotérica, ineficaz. Además, paradójicamente, la función de investigación no es atendida adecuadamente dentro de la institución universitaria, pues aunque su importancia se deduce de aspectos como la evaluación académica de los profesores, de su valor para las contrataciones y en los privilegios asociados a la condición de investigador, por otra parte se le tiene como prescindible con respecto a la función de docencia. La investigación es el rubro que primero y más se recorta dentro de las ya eternas crisis presupuestarias de las instituciones (Villarroel, 1990), crisis fuertemente agravada en la actualidad (García Larralde, 2011; Koudeir, 2019). Hoy en día, ya casi terminada la segunda década del siglo XXI, la investigación como función que de manera particular y especial atañe a las cátedras universitarias como células fundamentales, se encuentra en la práctica tenida como voluntaria, si acaso no abandonada.

En cuanto a la finalidad principal que en la práctica ha tenido la universidad venezolana, ella ha sido y continúa siendo eminentemente profesionista, no de investigación. Ha sido formadora de profesionales, y en ese sentido ha cumplido bien su función principal, aunque no sea para la producción sino para la certificación y confirmación de roles profesionales para la sociedad, con un profesional que no crea ni transforma, sino que mantiene y conserva (Albornoz, 2006). De modo que en Venezuela la universidad para el cambio y la transformación social, de influencias positivistas de finales del siglo XIX, y de la Reforma de Córdoba de buena parte del siglo XX, 
parece haberse quedado en el plano de las proclamas y las declaraciones, como un centro de poder simbólico, frecuentemente articulado al campo de la lucha de clases y de la contradicción ideológica (Villarroel, 1990). Esta universidad ha tenido rasgos de liberal hasta 1958 y luego gradualmente populista hasta llegar al siglo XXI, con la denominada Revolución Bolivariana, etapa en la cual ha sido, en el mejor de los casos, dejada a su suerte y otras veces atacada (Albornoz, 2006, 1999; Calatrava, 2016), donde es difícil afirmar qué tipo de universidad se tiene hoy en día, probablemente una institución bastante más que populista. La universidad venezolana de marcado acento liberal se mantuvo aproximadamente hasta hace unas seis décadas, cuando adquirió mayor democratización, con masificación estudiantil y búsqueda de justicia social. A fines de los 1960, tras el fenómeno de la Renovación Universitaria, la institución asumió un carácter más populista, con formación "crítica". Ya para la década de los 1980 y comienzos de los 1990 a la universidad venezolana se le trató de enrumbar por la vía de un modelo más tecnocrático, aunque en las universidades autónomas tendió a prevalecer el modelo populista (Albornoz, 1999; Parra-Sandoval, 2008; Villarroel, 1990). Tras la llegada de la era del chavismo en febrero de 1999, la vieja oposición entre universidad y Estado se ha reasumido, aunque de manera diferente. Es ahora la universidad autónoma la que defiende la democracia liberal ante un Estado que pretende imponer el denominado socialismo del siglo XXI a la educación y a la sociedad venezolana (Calatrava, 2016; Ramírez, 2011; Ramírez y Salcedo, 2016).

\section{RASGOS DE LA UNIVERSIDAD PÚBLICA VENEZOLANA CONTEMPORÁNEA.}

Los aspectos señalados anteriormente se relacionan con rasgos de la dirección o gerencia que han tenido las instituciones universitarias venezolanas en décadas más recientes (Rivas, 2014). Un trabajo que no se puede dejar de referir es el de Esté (1998), quien analizó a fines del siglo XX un marco temporal de aproximadamente tres décadas, lapso caracterizado por acelerada expansión del subsistema de educación superior y acuerdo entre las principales fuerzas políticas y sociales acerca de un proyecto democratizador, con altamente predominante responsabilidad financiera del Estado venezolano. En aquel período algunas reformas y ajustes se habían aplicado "sobre la marcha, en forma poco sistemática y poco planificada" (p. 135). Los ajustes implicaron, entre otras medidas, diversificación institucional y académica, regulación del crecimiento matricular y mayor centralización y control político de algunas instituciones. El complejo tejido del nivel superior de la educación venezolana ya representaba para la época un gran peso financiero para el Estado, esfuerzo que para fines de los 1990 no se había traducido en aumento del rendimiento académico ni de la eficiencia institucional. Más bien, en general la estrategia acometida trajo efectos negativos para el subsistema y la nación. Con todos los logros que pudiere haber, se cosechó un complejo de problemas de gestión y administración en un numeroso conjunto de instituciones, desarticuladas e internamente heterogéneas, con evidentes fallas tanto del modelo autonómico tradicional como del 
más reciente modelo dependiente, es decir, aquellas instituciones estatales fundadas a mediados de los 1970. Otros rasgos eran: insuficiente o inexistente desarrollo de métodos de evaluación interna, falta de racionalización de los recursos, baja productividad institucional, escasez de innovaciones académicas y organizativas, deficientes procesos de selección y evaluación del personal, fallas en el sistema de control y evaluación curricular, marginalidad de la investigación como función académica específica, ausencia de sistemas meritocráticos, falta de planificación, rígida estructura de gastos, enfoque clientelista, exagerada expansión del personal y aparato administrativo burocratizado e ineficiente. Todas estas habían sido las más resaltantes falencias de la gestión del subsistema de educación superior posterior a la Renovación Universitaria de comienzos de los 1970 (Esté, 1998), fallas que hoy en día están lejos de haberse subsanado ya casi finalizada la segunda década del siglo XXI.

Dentro de tal estado de cosas, con la crisis presupuestaria como fondo, para fines del siglo XX se había producido una desarticulación institucional, con solapes de funciones, de objetivos y desaprovechamiento de esfuerzos, experiencias y recursos desde arriba hacia abajo en la institución. Se generó falta de orientación en objetivos generales y específicos en todos los niveles institucionales, ambigüedad en concepciones, con una de acento liberal populista y otra con inclinaciones tecnocráticas. Tales concepciones derivaban de valores sociales diferentes, por una parte, la exaltación de lealtades y solidaridad social no siempre bien entendidas o aplicadas. Por la otra, pragmatismo social e individualismo. Con lo anterior, no sorprende que existiera bajo rendimiento institucional, tanto en la gestión como en la calidad académica, problemática que es interpretable por lo menos desde dos grandes perspectivas. Una, la que busca explicación en las bases estructurales del sistema social, lo cual implica crítica al contenido y la concepción educativa subyacente a la estrategia estatal. Otra, la que remite a causas inherentes al funcionamiento de las instituciones propiamente y al comportamiento de sus actores, por lo que la crítica va dirigida a la forma de instrumentación de la estrategia, desviada de sus objetivos originales. Entonces, ya para finales de los 1990 el sistema de educación superior venezolano se hallaba en una prolongada crisis de indefinición, con grave pérdida del dinamismo y potencial necesarios como centro estratégico de la sociedad y de la nación para impulsar el desarrollo a través de la innovación científica y tecnológica. Todo dentro del marco global del tipo de gestión pública que se había extendido a todos los niveles institucionales del país y sin que tampoco fueran generados cambios desde los actores internos de las instituciones universitarias (Esté, 1998).

Por otra parte, Corredor (1999) evidenció que en las universidades venezolanas no se formulaban metas institucionales desde la década de los 1970, lo que las colocaba, de cara al siglo XXI, "sin visión estratégica" y sin proyecto en torno a metas factibles de lograr, enmarcadas en 
propósitos de excelencia en las funciones de docencia, investigación y extensión. En este país, las metas institucionales se establecen por ley y se definen operativamente por reglamentos, decretos y resoluciones promulgados por decisión corporativa del gobierno universitario. Las metas definen la actividad interna de un proceso o de una organización y sirven como base para formular las políticas, para ser categoría específica del proceso planificador y para servir como referencia al proceso administrativo. Por tanto, el establecimiento de metas precede y preside el proceso planificador, todo ello idealmente en el marco de la Constitución Nacional, la Ley de Educación y la Ley de Universidades, según las cuales la universidad venezolana es una en toda la nación y resume metas tales como: servicio público del Estado, factor de producción, agente de cambio político, de eficiencia administrativa y formación profesional. Ello sin menoscabo de que en el devenir sociohistórico puedan plantearse discusiones acerca de la renovación de estructuras institucionales y redefinición de la misión y las metas (Corredor, 1999).

Para las universidades venezolanas existen metas generales y específicas, contenidas en reglamentos, resoluciones y otros instrumentos administrativos, tales como normas e instructivos universitarios. Para finales del siglo XX, la Universidad Central de Venezuela cumplía apenas medianamente sus metas y cuando lo hacía era en forma tradicional y con poca motivación. Existía una universidad desfasada, pues lo que hacía no es lo que el individuo y la sociedad necesitan. Una consecuencia de esto es que con frecuencia las empresas y otras organizaciones se ocupan en formar a sus líderes, y los egresados universitarios suelen seguir cursos complementarios para su mejor inserción en el mercado laboral. Existía bajo rendimiento académico y poca importancia otorgada al cumplimiento de la función administrativa, especialmente en lo concerniente a la disciplina en el trabajo y al proceso planificado de desarrollo. Adicionalmente, las normas y la planificación muy poco se cumplían. Es de resaltar que en la actualidad son muy pocos los estudios actualizados en el particular, aunque lo más probable es que los hallazgos de Corredor (1999), referidos a la más grande y antigua universidad del país, hallazgos muy coincidentes con los de Esté (1998) para con varias otras instituciones, sean en el mejor de los casos similares para el momento actual.

Por otro lado, García Guadilla (1998) ha sostenido que en cuanto a reformas en las instituciones de educación superior de Venezuela, había existido una Comisión de Evaluación Institucional de las Universidades desde 1983, instaurada por el Consejo Nacional de Universidades, la cual produjo orientaciones en la concepción, principios y criterios del sistema, así como un conjunto de indicadores cualitativos y cuantitativos. Para 1992 se había incorporado otro conjunto de indicadores, pero no se estaba considerando un sistema de evaluación institucional que privilegiara la evaluación orientada hacia el cambio. Ese tipo de evaluación no había tenido los resultados esperados, probablemente debido a que se concibió de manera poco participativa de las instancias 
a ser evaluadas y porque pocas universidades asumieron los compromisos establecidos. Para entonces algunas universidades públicas estaban adelantando propuestas de autoevaluación para estimular la participación y el compromiso de la comunidad académica. En cuanto a políticas de actualización académica, se declaraba la existencia de programas de generación de relevo en varias universidades estatales, con buenos resultados en algunas y con deseo de extenderlos a otras. En cuanto a innovaciones, destacó el desarrollo de Parques Tecnológicos por medio de asociaciones entre el Ejecutivo Nacional y algunas universidades, así como de empresas universitarias. En cuanto a vinculación entre universidad y comunidad, se encontró una gran actividad en educación ambiental, con iniciativas curriculares en pro de la conciencia ambiental, además de postgrados en esta área (García Guadilla, 1998).

En cuanto a innovación en sistemas de información, existía el sistema LUIS, por parte de la Biblioteca Nacional, para alumnos de pregrado y postgrado del país. También, la incorporación de las bibliotecas de las principales universidades nacionales a redes internacionales, así como la Red de la Biblioteca de la Universidad Central de Venezuela. En cuanto a organismos nacionales de coordinación de la Educación Superior, se hallaban el Consejo Nacional de Universidades, la Oficina de Planificación del Sector Universitario (OPSU) y la Dirección General Sectorial de Educación Superior del Ministerio de Educación. En lo referente a organismos de coordinación de información estadística, se contaba con la misma OPSU (García Guadilla, 1998), todo lo anterior sin embargo antes del inicio de la presente etapa de dos décadas del siglo XXI en el país, período en el cual lo que se venía ejecutando ha venido siendo alterado en una dirección muy diferente, contraria (Calatrava, 2016; Koudeir, 2019). Adicionalmente, García Guadilla (1998) señaló que en Venezuela las asociaciones gremiales habían jugado un papel muy importante en las reivindicaciones de sus afiliados en las universidades, pero con aumento de relaciones expresadas en el clientelismo partidista, el afianzamiento de lealtades y el sostén electoral de algunos que, por méritos ajenos a la academia pero cercanos a componendas, logran alcanzar posiciones de dirigencia. Eran gremios y asociaciones cuya filosofía y objetivos estaban siendo superados por la nueva realidad del país, aunque tal viejo estilo de dirigencia sindical continuaba teniendo peso en tanto que eran los únicos interlocutores organizados. Sin embargo, para entonces se observaban algunos cambios dentro del mundo profesoral, como la constitución de formas de asociación de prevaleciente orientación académica (García Guadilla, 1998).

Sumado a lo anterior, Albornoz (2006) ha expresado planteamientos críticos similares a los de Corredor (1999), Esté (1998), Morles (1998) y Villarroel (1990), aunque ha limitado principalmente su tratamiento a los modelos de universidad latinoamericana en general y venezolana en particular. Sostiene Albornoz (2006) que de lo que se trata es de dos visiones del desarrollo, uno 
ligado a la modernidad europea y el capitalismo avanzado (de Davos, Suiza) cercano al modelo pragmático de universidad, y otro ligado específicamente a la historia y la cultura de la América conquistada por españoles y portugueses (de Porto Alegre, Brasil), más propio de la universidad tradicional latinoamericana, denominada crítica. Este segundo modelo universitario con frecuencia se corresponde con sectores que declaran no creer en el desarrollo propio del capitalismo avanzado originado en Europa noroccidental en el siglo XVII, pero que al parecer también quieren y buscan desarrollarse (sostienen que "un mundo mejor es posible") en un contexto como el de Venezuela, en donde se dice que existe el talento pero no los fondos materiales, ni la cultura organizacional académica ni la visión de futuro con apoyo estatal. Se trata de un país donde no existen o son muy precarios los cuatro polos del desarrollo académico, tales como la producción, la productividad, la difusión y el impacto (Albornoz, 2006).

Para el logro del declarado nuevo tipo de desarrollo, el modelo de Porto Alegre supone cambios sociales radicales a fin de mejorar la calidad de vida en todas sus instancias, pero descuida aspectos del modelo de Davos, como lo son los componentes éticos (el trabajo honesto, el "factor decencia" o el comportamiento ético en sociedad), el sentido de ciudadanía y la responsabilidad individual, así como la cohesión social e institucional. La propuesta de Porto Alegre muestra una retórica en pro de mejoras sociales, pero por otra parte ejecuta una praxis anti-desarrollo, esto es, implementación de ideas "progresistas" que contradictoriamente producen atraso en la sociedad y sus organizaciones. Así, la universidad deseable en países como Venezuela debería hallarse entre los dos extremos, esto es, en la creación de una élite meritocrática, al mismo tiempo que se atienda al principio de ser una institución democrática y popular. Esta universidad deseable será una que tome decisiones en forma iterativa, progresiva, a menudo lenta y con frecuencia experimental; que se reforme aplicando análisis de evaluación institucional, diagnósticos, planificación y programación, evaluación de resultados y entrenamiento permanente de su comunidad (Albornoz, 2006).

Así, dentro del marco expresado, es pertinente señalar que la crítica, tanto desde adentro (De Venanzi, 1983; Losada Aldana, 1984; Mayz, 1984) como desde afuera, desde los gobiernos (Peñalver, 1983), acerca de la manera como estaba siendo conducida la universidad venezolana, tiene unos cuarenta años. Viene de entre fines de los 1970 y comienzos de los 1980, tras lo que parecieron dos décadas de una suerte de encantamiento romántico de buena parte de la ciudadanía y la élite cultural del país con respecto a sus universidades. Aquel lapso de dos décadas tras la caída de la dictadura perezjimenista en enero de 1958 al parecer había sido ya suficiente para que algunos consideraran a la universidad venezolana en su conjunto como "una entidad fuera de época, poco adaptada y con precarias respuestas a las exigencias del país" (Peñalver, 1983, p. 18), esto es, una entidad desfasada, ineficientemente gerenciada. Con relación a ello, unas dos décadas después, 
mirando a la universidad de finales del siglo XX y comienzos del XXI, Albornoz (1999) se refirió al advenimiento de nuevas realidades y nuevos paradigmas en la educación superior. Para esos años había prevalecido un discurso "unesquiano" de cierta burocracia internacional, la cual había buscado nuevos caminos interpretativos, con un enfoque conciliatorio que a veces pagaba tributo al falso consenso. No obstante, se habían tratado de aplicar nuevos paradigmas analíticos que dejaran atrás la noción de la universidad tradicional latinoamericana como fundamentalmente un lugar de empleo de por vida, cuya gerencia había sido básicamente el manejo del personal o de una nómina de pago. En un ambiente de "clientelismo perverso", en nuestras universidades se había dado paso a algunas reformas, con frecuencia muy lentas, con algo de mayor adecuación de las instituciones al campo laboral y a demandas de la sociedad (Albornoz, 1999), situación en la cual la universidad venezolana ingresó rezagada al siglo XXI y hoy en día parece encontrarse aún más, en medio de nuevos y serios obstáculos (Koudeir, 2019).

\section{TIEMPOS UNIVERSITARIOS "BOLIVARIANOS".}

La era del chavismo advino en Venezuela en febrero de 1999. Con referencia a los tipos de universidad que existían en Venezuela antes de la denominada Revolución Bolivariana, Albornoz (1999) ha señalado que el único modelo de excelencia en el país ha sido el de la universidad estatal. Para aquel tiempo, según dicho autor, las universidades de investigación eran seis, todas por lo menos en parte influidas por el modelo norteamericano, que por esa vía se aproximaban indirectamente al alemán de Humboldt. Luego, las universidades de docencia eran cinco, mientras que las universidades "marginales al mundo académico" eran tres. Por otra parte, las numerosas instituciones restantes del país, tanto públicas como privadas, eran instituciones "sin clasificación" (Albornoz, 1999). Para entonces, cambios hacia las tendencia mundiales en educación superior parecían improbables, pues el segundo gobierno de Rafael Caldera (1994-1999) había desatendido al sector universitario nacional. Para el año 2020 el progreso luce aún más improbable, debido a que no solamente la educación universitaria, sino todo el sector de ciencia, tecnología e innovación del país marchan a contravía de las tendencias mundiales (Ramírez y Salcedo, 2016). Así, en ese ambiente institucional tan poco favorable, ya entrado el siglo XXI Cárdenas (2004) consideró que los principales obstáculos y desafíos de la universidad venezolana eran: falta de unidad institucional, sistemas administrativos no adaptados a las nuevas condiciones institucionales, falta de coordinación con otras instituciones de educación superior, currículos que no corresponden a las nuevas exigencias del humanismo y de la ciencia, falsa disyuntiva entre equidad y calidad en relación con el ingreso de nuevos estudiantes, precario desarrollo de la investigación y de los postgrados, falta de respuestas a las mayores y más complejas exigencias de la sociedad, cortoplacismo en la visión, falta de un sistema de jubilaciones y pensiones que no afecte el presupuesto ordinario, influencia del partidismo político y falta de un sistema de evaluación institucional. Para el futuro 
inmediato eran prioritarios la atención al pregrado, la formación ética, el uso de las nuevas tecnologías de la información y el conocimiento, la educación permanente, la diversificación de la educación superior, la evaluación y la acreditación, la internacionalización y el financiamiento (Cárdenas, 2004).

Por otra parte, Parra Sandoval (2008) se refirió a la especificidad de la universidad venezolana y su situación reciente. Según dicha autora, estas instituciones, tanto públicas como privadas, han funcionado tradicionalmente sometidas a constantes altibajos condicionados a la relación con el Estado y con la sociedad, donde el único vínculo importante entre las universidades y el Estado ha sido el financiamiento, en un contexto en el cual más recientemente han prevalecido tanto el desconocimiento como la descalificación mutuas. En cuanto a la relación de las universidades con la sociedad, esta, por lo menos hasta hace poco, había colocado en las altas casas de estudios sus expectativas de ascenso social, relacionadas con la condición de gratuidad del servicio educativo, por el cual hubo un crecimiento matricular sostenido, que para 2004 era de más de 600 mil estudiantes matriculados en instituciones públicas y privadas, ello sin incluir las más recientemente creadas, las gubernamentales Universidad Bolivariana, de las Fuerzas Armadas y la Misión Sucre, entre otras. Dicho crecimiento matricular ha incidido en la consolidación del modelo profesionalizante, con énfasis en la docencia y la formación de pregrado. Por otro lado, la tendencia en la selección de carreras se ha mantenido a favor de las de ciencias sociales y económicas, con menores porcentajes para carreras cortas y técnicas.

Con relación a la función de investigación, vale mencionar que ya entrado el siglo XXI, para 2004 menos del 4\% de los profesores universitarios del país participaron en la convocatoria del estatal Programa de Estímulo al Investigador, evidenciándose que en las universidades venezolanas tal función posee un status marginal. Esto se relaciona con el modelo híbrido de la universidad venezolana, el cual ha intentado combinar tres frentes: formación profesional, búsqueda de la verdad y compromiso social, entendiendo principalmente por esto último la identificación con necesidades populares y la vocación por lo político. La concepción profesionalizante de la universidad venezolana ha privilegiado la trasmisión de conocimientos por sobre su generación, y el grupo de $15 \%$ de instituciones consideradas grandes no muestra coincidencia lógica en cuanto a su aporte en investigación. El modo asumido en el país en cuanto a privilegiar la docencia reconocía como importante la investigación solo si expresaba compromiso social de la universidad, buscando resguardar la autonomía universitaria de cualquier intromisión, dejando frecuentemente a un lado la búsqueda de la verdad. Esta situación funcionó de manera más o menos inalterada hasta aproximadamente mediados de los 1990, cuando las universidades tuvieron que aceptar que les fijaran reglas de juego diferentes, por lo menos en cuanto al personal de investigación, dentro 
de un contexto internacional cada vez más altamente competitivo (Parra Sandoval, 2008) y luego ingresar en un territorio sumamente difícil en el siglo XXI (Vessuri, 2003).

De otro lado, con referencia a la calidad, la evaluación y la acreditación de las instituciones universitarias de Venezuela, Fuenmayor (s/f), en un libro publicado probablemente alrededor de 2009, enfatizó el esfuerzo que significó la creación e instrumentación a comienzos del siglo XXI del estatal Proyecto "Alma Mater" para el mejoramiento de la calidad y equidad de la educación universitaria de Venezuela, proyecto dentro del cual el Sistema de Evaluación y Acreditación (SEA) fue una iniciativa central. Según dicho autor, el Proyecto “Alma Mater” constituyó la expresión más acabada, más universal y de mayor impacto dentro de la política en educación superior que llevó a cabo el Estado venezolano por medio de la Oficina de Planificación del Sector Universitario (OPSU) entre febrero de 1999 y diciembre de 2004, ello bajo el lema de "Hacia la excelencia académica universitaria con equidad y pertinencia social". El proyecto SEA fue aprobado por unanimidad en el Consejo Nacional de Universidades como primer sistema de evaluación y acreditación universitaria de Venezuela, el cual perseguía el desarrollo de la academia, así como el mejoramiento constante de su calidad. Según el referido autor, dicho sistema se creó pero nunca se instrumentó (Fuenmayor (s/f), de lo cual se deduce que ya bien entrada la segunda década del siglo XXI, en la educación superior venezolana se presentaban serios problemas en cuanto al aseguramiento de la calidad de las instituciones universitarias del país, apreciación que coincide con planteamientos de Cárdenas (2004) y de Suárez Amaya y Díaz Barrios (2014).

Adicionalmente, según Delgado (2011), en Venezuela los esfuerzos por promover el acceso a la educación superior no son nuevos. Han sido continuos desde mediados de los 1950. Como ejemplo a la mano, el porcentaje del liderazgo nacional con título universitario aumentó en este país desde un 9\% en 1961 a $45 \%$ en 1985. Igualmente, la matrícula pasó de unos 20 mil estudiantes universitarios en 1960 a 500.000 en 1988. Para 1990 el cuerpo de instituciones de educación superior de Venezuela era de 28 universidades nacionales autónomas, 12 nacionales experimentales, 12 privadas y 63 instituciones no universitarias. Las universidades nacionales autónomas son las más antiguas, más prestigiosas y más grandes instituciones de educación superior del país, grupo que incluye a la Universidad Central de Venezuela, fundada en 1721, la Universidad de Los Andes, fundada en 1810, la Universidad del Zulia, en 1891 y la Universidad de Carabobo, en 1892. Estas universidades poseen, aunque cada vez menos, autonomía organizacional, académica, administrativa y financiera. Son regidas de manera corporativa en más de treinta diferentes niveles jerárquicos, con una estructura académica organizada en torno a facultades. Las universidades experimentales son instituciones creadas por el Ejecutivo Nacional a partir de los 1970 con la finalidad de, entre otras, experimentar con distintos enfoques académicos y administrativos en la 
educación superior. Estas instituciones poseen autonomía pero sus autoridades son designadas por el Ministro de Educación Superior (Delgado, 2011).

Dentro del sector público de Venezuela la educación superior ha sido gratuita, donde el Estado suministra cerca del 95\% del apoyo financiero y el resto proviene de ingresos como los de cursos de postgrado y extensión, así como de aranceles. Esto ha sido una suerte de espada de doble filo, pues las mejores instituciones, aquellas donde la investigación y los programas avanzados se llevan a cabo, dependen casi exclusivamente del presupuesto gubernamental y se encuentran a merced de los vaivenes de la economía y la política. Para 1994 la educación superior venezolana consumía el $43.6 \%$ del presupuesto nacional para educación y $15.3 \%$ del nacional. Para tal época, había 17 universidades públicas y 18 privadas. En 1972 existían 89 programas de postgrado en el país y para 1994 había 1.047 , de los cuales el 7\% ciento eran doctorales, 46\% de maestría y $47 \%$ de especialización. Para fines del siglo XX la Universidad Central de Venezuela conducía aproximadamente la tercera parte de todos los programas de postgrado del país (Delgado, 2011). Por su parte, para Graffe (2006), en Venezuela desde hace mucho tiempo se ha evidenciado una tendencia hacia la expansión de la educación superior, con prevalencia del sector estatal en términos de absorción de la demanda, dentro de una concepción binaria por la cual se cuenta con un alto número de instituciones universitarias y no universitarias. El Sistema de Educación Universitaria de la República Bolivariana de Venezuela ha venido siendo esencialmente un sistema grande de educación pública, con rasgos de privatización, caracterizado por el acceso de masas y donde las universidades asumen una carga significativa de la matrícula, con un mayor énfasis en la formación en ciencias sociales, que incluyen a las ciencias económicas y las administrativas. En segundo lugar, la mayor dedicación es hacia la enseñanza de las disciplinas de la ingeniería, arquitectura y tecnología (Graffe, 2006). El anterior escenario en parte se ha modificado más recientemente por varias razones, entre ellas por la creación de un grupo de nuevas instituciones de educación superior en varias regiones del país, así como las denominadas Misiones, a lo largo de lo que va del siglo XXI, lo cual ha dado lugar a preocupaciones acerca de la falta de calidad del servicio educativo (Calatrava, 2016; Fuenmayor, s/f).

Por otra parte, los resultados del estudio de Suárez Amaya y Díaz Barrios (2014) con referencia a las cuatro universidades venezolanas con mejor posicionamiento global en investigación, arrojaron características que en la mayoría de los casos conspiran en contra de una gestión sistémica, integral e integradora. Sin embargo, se observó mayor flexibilidad y facilidad de cambio en la estatal Universidad Simón Bolívar, con un modelo académico-organizacional por departamentos, descentralizado en las decisiones, con fuerte cultura de investigación y sin mayores obstáculos culturales en cuanto a ser evaluada. Estas características le otorgan mayores 
posibilidades de acercarse a una universidad innovadora, frente a otras universidades tradicionales como la Universidad de Los Andes, la Universidad Central de Venezuela y la Universidad del Zulia, burocráticas, centralizadas, con una cultura orientada hacia la docencia y con fuertes obstáculos culturales en cuanto a ser evaluadas.

No obstante, se encontraron prácticas de gestión en cada universidad que las apuntalan con referencia a investigación y que explican su relativamente buena posición en Latinoamérica. Si bien la Universidad Simón Bolívar se distingue de las otras tres universidades, estas también han desarrollado capacidades que deben ser consideradas, como es el caso de la Universidad Central de Venezuela, que cuenta con visibilidad en clasificaciones latinoamericanas debido a la productividad científica de investigadores de trayectoria, además de contar con una fundación que favorece relaciones con el sector productivo.

En lo que respecta a la Universidad de Los Andes, ella cuenta con programas internos de estímulo a la investigación, así como una página web de avanzada con acceso abierto que le ha permitido proyectar su producción científica, a sus investigadores y ganar mayor visibilidad. Y la Universidad del Zulia desarrolló como un valor la pertenencia al Programa de Promoción del Investigador (ya para 2020 inexistente en el país), lo cual ha permitido lograr una cantidad importante de investigadores noveles que son la continuidad de la investigación en el largo plazo. Partiendo de que las cuatro universidades estudiadas por Suárez Amaya y Díaz Barrios (2014) poseen aspectos positivos que les otorgan liderazgo de investigación en el país, la pregunta es ¿por qué la Universidad Simón Bolívar descuella en primer lugar?

Además de ser considerada joven y pequeña en comparación con las otras tres (todas centenarias y con más de dos mil profesores cada una), hubo dos aspectos de la gestión que definitivamente hacen diferencia: su estructura dinámica-flexible y su cultura. Una estructura más plana y descentralizada promueve, por una parte, el ingreso y permanencia de profesores en investigación y, por otra, la integración de recursos y servicios favorece la vinculación con el sector productivo y la generación de ingresos para investigación. En cuanto a la cultura, se promueve la permanencia del académico en el área de investigación al haberla desarrollado como un valor. Adicionalmente, se observaron otros dos elementos de la gestión (planificación y control) que están en desarrollo y potencialmente deben convertirse en elementos diferenciadores si las otras universidades no cambian. Por un lado, la Simón Bolívar tiene una actitud proclive a la planificación como herramienta para la acción, desarrolla objetivos específicos cuantificados en el área operativa los cuales son susceptibles de control, además de haber desarrollado indicadores para evaluar la gestión. Ambos elementos la colocan en el camino de tener mejor control de su 
gestión (Suárez Amaya y Díaz Barrios, 2014).

Ahora, en medio de la muy severa crisis actual, los anteriores aspectos constituyen capacidades distintivas reales y potenciales para el mayor desarrollo de investigadores, favorecer la obtención de recursos aparte de los tradicionales, aumentar la productividad científica y la visibilidad de la institución en el ámbito internacional. Esto puede promover un modelo de desarrollo con visión realista, que exprese la universidad que se busca lograr, en función de recursos y capacidades. Pero al igual que las otras tres instituciones estudiadas, la Universidad Simón Bolívar también presenta debilidades que deberá superar. Podría decirse que estas cuatro universidades presentan atributos que las convierten en líderes en investigación en el país, y su buen posicionamiento las ha llevado a una buena presencia en la comunidad académica regional.

No obstante, presentan falencias que son impedimentos para el desarrollo de la investigación. Así, de todo lo anterior se pueden derivar algunas interpretaciones: por un lado, tal como antes lo han evidenciado Esté (1998), Albornoz (1999) y Corredor (1999), las universidades tradicionales venezolanas tienen un modelo de gestión agotado que requiere cambios profundos si de verdad se busca elevar el nivel de las instituciones para sostener su existencia a largo plazo. Por otro lado, puede decirse que las universidades públicas venezolanas presentan particularidades y fortalezas que podrían ayudarlas a llevar a cabo una gestión basada en las mejores prácticas de cada una. La sinergia de recursos, procesos y experiencias podría elevar el nivel de la investigación en todas las universidades del país, de manera que no sean solamente cuatro las que se encuentren en las clasificaciones latinoamericanas, sino muchas más instituciones (Suárez Amaya y Díaz Barrios, 2014).

Más recientemente, Koudeir (2019) ha señalado que el mayor crecimiento de las universidades venezolanas ocurrió junto con el desgaste del sistema político de la democracia representativa post 1958. Así, en 1999 asumió el poder el Teniente Coronel Hugo Chávez, cuyo gobierno otorgó rango constitucional a la autonomía universitaria, se reconocieron deudas incumplidas al sector universitario desde los 1970 y se aprobó en 2005 la Ley Orgánica de Ciencia, Tecnología e Innovación para ambiciosos proyectos en dicho ámbito. Hoy, tras más de 20 años de la denominada Revolución Bolivariana, de fuerte influencia castrista, la situación de las universidades venezolanas es penosa. Desde 2003 se han ignorado las necesidades de las instituciones, se ha vivido una fuerte caída de la producción científica nacional y un deterioro grave del funcionamiento de las altas casas de estudio.

Un informe reciente del Observatorio de Derechos Humanos de la Universidad de los Andes, 
titulado El sistema paralelo universitario en Venezuela muestra la situación universitaria del país, con la aparición de las misiones paralelas Sucre y Alma Mater. De 61 universidades públicas, 52 están controladas por el gobierno y en los últimos 20 años se han creado por lo menos 38 instituciones universitarias casi exclusivamente de docencia, caracterizadas por nombramientos de autoridades frecuentemente sin credenciales académicas y contratación de personal sin lo necesario para la actividad de docencia e investigación. En estas instituciones la vinculación con el partido oficialista prima sobre cualquier intento por mejorar la actividad universitaria (Koudeir, 2019).

Como otro dato importante, entre los años 2004 y 2017 las universidades no controladas por el gobierno recibieron un aproximado de 68 millones de dólares para sus varias funciones intrínsecas, mientras que al otro sistema paralelo se le otorgaron casi 400 millones, los cuales son mayormente utilizados en actividades de política partidista. Con el empeoramiento de la crisis económica en los últimos años con Nicolás Maduro y la situación del sector oficial de la educación superior, los resultados no parecen justificar tales erogaciones en instituciones paralelas. La partidización y la ideologización en una doctrina ortodoxa que no tiene que ver con la "comunidad de intereses espirituales que reúne a profesores y estudiantes en la tarea de buscar la verdad y afianzar los valores trascendentales del hombre" que señala la Ley de Universidades, han conducido a la entronización de un sistema universitario de baja calidad.

Más recientemente, en 2019, ha sido publicada una sentencia de la Sala Electoral del abiertamente pro gubernamental Tribunal Supremo de Justicia, la cual constituye un intento por ahogar la autonomía universitaria que aún pudiera existir. Dicha decisión judicial usurpa atribuciones expresamente garantizadas por la Constitución Nacional a las universidades y muestra intenciones poco democráticas para con las universidades autónomas (Koudeir, 2019).

\section{CONCLUSIONES}

En primer lugar, se puede concluir que la revisión de la literatura más reciente acerca del tópico abordado avala la teoría que sostiene que la universidad estatal venezolana, en el marco de la universidad tradicional latinoamericana, ha sido una institución fundamentalmente profesionista, mayormente volcada hacia la docencia y en la cual la investigación es, la mayor parte de las veces, marginal. También, que es una institución altamente dependiente, pero a la vez enfrentada con el Estado y los sucesivos gobiernos. Adicionalmente, es una universidad que ha sufrido y sufre de serias fallas en su gestión interna, las cuales ahora en el tan difícil escenario de las primeras décadas del siglo XXI parecen mucho más difíciles de enfrentar y corregir. En segundo lugar, las universidades venezolanas no son torres de marfil aisladas, sino que han estado y están integradas a lo cotidiano de las actividades políticas y sociales. Este país no solamente ingresó tarde al fenómeno 
universitario de Hispanoamérica, sino que su entrada al siglo XX fue también tardía y en el siglo XXI al parecer se halla igualmente demorada. A partir de la caída de la dictadura del general Marcos Pérez Jiménez en 1958 se remozaron las instituciones universitarias y luego de 1970 las principales universidades estatales autónomas mostraban su mayor vigor, con postgrados de investigación científica, humanística y tecnológica. En esa década las universidades venezolanas se masificaron, creció el ingreso de estudiantes, pero sin el necesario complemento de profesores investigadores, y aumentó la burocracia en la administración universitaria, lo cual es hasta hoy un serio problema. Por otro lado, se agudizaron los inconvenientes derivados de la intromisión del partidismo político en los asuntos académicos, sin que se hayan aplicado medidas correctivas, sobre todo en las instituciones directamente dependientes del gobierno. Las ideas de reforma presentadas por, entre otros, connotados académicos venezolanos como Ernesto Mayz Vallenilla y Francisco De Venanzi en los 1980 se quedaron en debates y propuestas.

Pero con todo, en la década de los 1990 hubo avances y mejoras, tales como el incentivo y reconocimiento por parte del Estado a los investigadores por medio del Sistema de Promoción al Investigador, además de proyectos con relación a políticas públicas, por parte del sector público y el privado. En esa década, de tímidos acercamientos hacia el modelo de la universidad pragmática del capitalismo avanzado, las universidades obtuvieron mayor apoyo financiero para la investigación, se consolidaron numerosos postgrados y se crearon otros, acreditados por el Consejo Nacional de Universidades. La primera creación y aplicación de internet fue en una universidad estatal autónoma y tuvo lugar la participación de estas instituciones en ámbitos como la industria petrolera y el sector de la salud. Los logros de esa década permearon hasta los primeros años del siglo XXI cuando, tras la llegada al poder de la denominada Revolución Bolivariana en 1999, el estado de cosas comenzó a cambiar.

Se dio inicio a un importante programa de acreditación de la calidad de las instituciones (Proyecto Alma Mater) pero a los pocos años fue suspendido y hoy en día ya no existe. En los últimos diez años aproximadamente la educación universitaria venezolana tal como se le conoció a finales del siglo XX lo que ha hecho es retroceder, declive que es parte del desplome del sistema nacional de transmisión y generación de conocimientos, de ciencia, tecnología e innovación del país. En consecuencia, salir de la dirección de contravía que actualmente se lleva en el ámbito de la educación superior venezolana parece una necesidad impostergable. Pero tal cambio tiene que ver con un asunto político de envergadura sobre el cual no tiene control la universidad. 


\section{REFERENCIAS BIBLIOGRÁFICAS}

Acevedo Tarazona, A. \& Correa Lugos, A. (2018). Un siglo del Manifiesto Liminar: Acción política y rebeldía en defensa de la universidad colombiana. Historia de la Educación Latinoamericana. 20 (30), 53-66.

Aguado-López, E. \& Becerril García, A. (2016). Producción científica venezolana: Apuntes sobre su pérdida de liderazgo en Latinoamérica. Revista Venezolana de Gerencia. 21 (73), 11-29. Disponible en https://www.redalyc.org/pdf/290/29045347002.pdf Acceso el 26 de julio de 2019

Aguirre Lora, M.E. (1997). Presentación a la edición en español. En: Bonvecchio, C. El mito de la universidad. Séptima edición. México, D.F.: Siglo Veintiuno.

Albornoz, O. (2006). La universidad latinoamericana. Entre Davos y Porto Alegre. Caracas: Los Libros de El Nacional.

Albornoz, O. (1999). Del fraude a la estafa. La educación en Venezuela. Caracas: Facultad de Ciencias Económicas y Sociales. Universidad Central de Venezuela.

Bifano, C. (2009). Investigación, postgrado y docencia de pregrado: eje académico de la universidad. En: Seminario Internacional Autonomía Universitaria: Compromiso Académico y Social. (pp.159-195). Caracas: Vicerrectorado Académico y Centro de Estudios de América. Universidad Central de Venezuela.

Bifano, J. (2008). Luces entre sombras. La UCV, el CDCH y la investigación universitaria. Caracas: Consejo de Desarrollo Científico y Humanístico. Universidad Central de Venezuela.

Blanco, C.E. (2007). Discurso y conocimiento en la investigación educativa. Caracas: Vicerrectorado Académico y Consejo de Desarrollo Científico y Humanístico. Universidad Central de Venezuela.

Calatrava, C. (2016). La máscara rota. Análisis de la intervención del gobierno de Hugo Chávez en el sistema educativo venezolano. Caracas: Universidad Católica Andrés Bello.

Cárdenas, A. (2004). El concepto de universidad. Origen y evolución. Mérida, Venezuela: Universidad de Los Andes. 
Corredor, J. (1999). Las metas de la universidad. Una propuesta de transformación. Caracas: Consejo de Desarrollo Científico y Humanístico. Universidad Central de Venezuela.

Delgado, J. (2011). Journal publication in Chile, Colombia and Venezuela: University responses to global, regional, and national pressures and trends. Unpublished doctoral dissertation. University of Pittsburgh. School of Education. Pittsburgh, PA, USA. Disponible en http://revistacts. net/files/Jorge_Delgado_Dissertation_ETD_08192011.pdf Acceso el 16 de agosto de 2019

De Venanzi, F. (1983). Respuesta al Dr. Mayz Vallenilla. En: Di Prisco, C. \& Wagner, E. (1990). Investigación y docencia en las universidades. (pp. 40-43). Caracas: Fondo Editorial Acta Científica Venezolana.

Esté, N. (1998). La educación superior venezolana. Una institución en crisis. Caracas: Consejo de Desarrollo Científico y Humanístico. Universidad Central de Venezuela.

Fuenmayor, L. (s/f). Prólogo. En: Villarroel, C. La acreditación universitaria: Una ilusión de la calidad. (pp. vii-xiii). Caracas: Consejo Nacional de Universidades-Oficina para la Planificación del Sector Universitario.

García Guadilla, C. (1998). Situación y principales dinámicas de transformación de la Educación Superior en América Latina. Caracas: IESALC-UNESCO-FUNDAYACHUCHO.

García Larralde, H. (2011). ¿Es viable el actual modelo financiero de las universidades? En: La universidad venezolana en el siglo XXI. (pp.164-178). Caracas: Universidad Católica Andrés Bello.

Graffe, G. (2006). Cobertura de la educación superior de pregrado en los años noventa. Estudio comparado: Colombia y Venezuela. Agenda Académica. 13 (1-2), 9-24.

Inciarte, A., García Guadilla, C. \& Rama, C. (2011). Líneas de transformación para la universidad latinoamericana en el siglo XXI. En: La universidad venezolana en el siglo XXI. (pp.199-248). Caracas: Universidad Católica Andrés Bello.

Koudeir, A. (2019). Universidades y ciencia en Venezuela. Disponible en https://revistapersea. com/ciencia-sociedad/universidades-y-ciencia-en-venezuela/ Acceso el 02 de febrero de 2020. 
LEY DE UNIVERSIDADES. (1970). Congreso de la República de Venezuela. Caracas: Venezuela. Gaceta Oficial Extraordinaria del 8 de septiembre.

Losada Aldana, R. (1984). Investigación universitaria contra subdesarrollo. Caracas: Consejo de Profesores Universitarios Jubilados. Universidad Central de Venezuela.

Mayz Vallenilla, E. (1984). El ocaso de las universidades. Caracas: Monte Ávila.

Mendoza Angulo, J. (2011). Sobre la especificidad de la universidad: Algunas peculiaridades atinentes a la naturaleza de la universidad autónoma. En: La universidad venezolana en el siglo XXI. (pp.149-160). Caracas: Universidad Católica Andrés Bello.

Morles, V. (1998). La universidad latinoamericana actual. Necesidad de replantear su misión. Revista de Pedagogía. 19 (56), 7-21.

Parra Sandoval, M.C. (2008). Las intimidades de la academia. Un estudio cuanti-cualitativo sobre la dinámica de la profesión académica. Maracaibo, Venezuela: Vice Rectorado Académico. Universidad del Zulia.

Peñalver, L. (1983). Prólogo. En: Tunnermann, C. La reforma universitaria de Córdoba. (pp. 9-22). Caracas: FEDES.

Ramírez, T. (2011). Democracia política, democracia académica y democracia universitaria. En: La universidad venezolana en el siglo XXI. (pp.109-117) Caracas: Universidad Católica Andrés Bello.

Ramírez. T. \& Salcedo, A. (2016). Inversión y producción científica en Venezuela. ¿Una relación inversamente proporcional? Revista de Pedagogía. 37 (101), 147-174 Disponible en http:// www.redalyc.org/pdf/659/65950543008.pdf Acceso el 18 de diciembre de 2019

Rivas, G. (2014). La gerencia de la educación universitaria en la sociedad del conocimiento. TELOS. 16 (3), 373-387 Disponible en http://ojs.urbe.edu/index.php/telos/article/view/2225/2072 Acceso el 14 de diciembre de 2019

Tunnermann, C. (1983). La reforma universitaria de Córdoba. Caracas: FEDES. 
Van Noorden, R. (2014). South America by the numbers. Nature. 510. 202-203.

Vessuri, H. (2003). Entre papers, probetas y probanzas. Tomo II. En: Baptista, A. (Coord). Venezuela Siglo XXI: Visiones y Testimonios. (pp. 79-106). Caracas: Fundación Polar.

Villarroel, C. (1990). La universidad y su productividad académica: Crítica y perspectiva. Caracas: Dolvia. 